\title{
Differential Second Primary Malignancy Occurrence After Breast Cancer According to HER2 Status: A Population-Based Study
}

\author{
Xiaoyi $\operatorname{Lin} \mathbb{D}^{1,2, *}$ \\ Xin $\operatorname{Lin}^{1,3, *}$ \\ Yingzi Li (iD) 1,2,* \\ Yuchen Zhang (D) 1,4 \\ Jiali Lin (D) \\ Guochun Zhang iD ' \\ 'Department of Breast Cancer, \\ Guangdong Provincial People's Hospital, \\ Guangdong Academy of Medical Sciences, \\ Guangzhou, Guangdong, People's \\ Republic of China; ${ }^{2}$ Shantou University \\ Medical College, Shantou, Guangdong, \\ People's Republic of China; ${ }^{3}$ The Second \\ School of Clinical Medicine, Southern \\ Medical University, Guangzhou, \\ Guangdong, People's Republic of China; \\ ${ }^{4}$ School of Medicine, South China \\ University of Technology, Guangzhou, \\ Guangdong, People's Republic of China \\ *These authors contributed equally to \\ this work
}

Correspondence: Guochun Zhang Department of Breast Cancer, Guangdong Provincial People's Hospital, Guangdong Academy of Medical Sciences, 106 Zhongshan Er Road, Yuexiu District, Guangzhou, 510080, Guangdong, People's Republic of China

Tel/Fax +86 20-838278I2-509/0

Email zhangguochun@gdph.org.cn
Purpose: Our study aims to analyze the association between HER2 status of breast cancer (BC) and second primary malignancy (SPM) occurrence.

Materials and Methods: Based on BC patients registered between 2010 and 2018 in the NCI SEER database, we utilized standardized incidence ratio (SIR) and Poisson regression to quantify SPM occurrence compared with the general population. Then, adjusted for competing death risk, cumulative incidence function and Gray's test were adopted to estimate the probability of SPM. Subsequent proportional subdistribution hazards regression was executed to identify the HER2 status impact on SPM risk. Finally, survival analysis was performed.

Results: A total of 409,796 first BC patients were included and 18,283 were identified with at least one SPM. The SIR of SPM after HER2+ BC was significantly lower than HER2- BC (1.03 vs 1.13 ; RR, 0.92; 95\% CI, 0.88-0.96; $\mathrm{p}<0.001$ ). The predominantly declining SPM risk was only observed for second $\mathrm{BC}(\mathrm{RR}, 0.89 ; 95 \% \mathrm{CI}, 0.82-0.96 ; \mathrm{p}=0.003)$ and lung cancer $(\mathrm{RR}, 0.84 ; 95 \% \mathrm{CI}, 0.74-0.95 ; \mathrm{p}=0.007)$. Furthermore, competing risk analysis verified the protective effect of HER2 positivity status on SPM occurrence. The 5-year cumulative incidence of SPM following HER2+ and HER2- BC were $4.09 \%$ and $5.16 \%$, respectively $(\mathrm{p}<0.001)$. In addition, among patients suffering from SPM, HER2 positivity status contributed to better overall survival.

Conclusion: It is demonstrated that HER2+ BC patients had lower SPM incidence, which was remarkable for second $\mathrm{BC}$ and lung cancer.

Keywords: breast cancer, HER2, second primary malignancy, incidence, SEER

\section{Introduction}

Breast cancer (BC) has become the most common cancer in women, and the leading cause of cancer death. ${ }^{1}$ During the past decades, dramatic survival benefit has been achieved by profound understanding of BC biology, widely applied early screening,

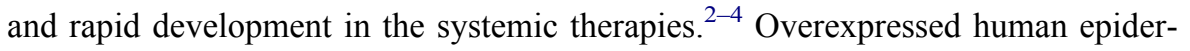
mal growth factor receptor 2 (HER2) was recognized as a major driver in 18-20\% of BC. 5,6 Fortunately, anti-HER2 therapies represented by trastuzumab have become the standard treatment for HER2-positive (HER2+) BC patients, and substantially improved their prognosis. ${ }^{7-9}$ However, the favorable survival outcome renders $\mathrm{BC}$ patient a higher probability of second primary malignancy (SPM) occurrence. $^{10}$ A recent case-control study reported excess risk for SPM among BC survivors, with an adjusted standardized incidence ratio of $12.94 .^{11}$ 
Since SPM can lead to excess mortality in BC patients, ${ }^{12,13}$ SPM occurrence and its risk factors raised the awareness of clinicians. In previous research, SPM risk decreased with age, ${ }^{14}$ with an exception of lung cancer that is aging-related. ${ }^{15}$ Negative hormone receptor was correlated with increased risk of SPM, ${ }^{16-18}$ which probably shared the same etiologic factors with first BC. Regarding postoperative treatment, the effect of chemotherapy remained controversial, ${ }^{18-20}$ although several literatures reached the agreement that utilization of DNAdamaging chemicals led to increased incidence of SPM. ${ }^{21}$ Radiotherapy may subject patients to a greater risk of SPM, especially lung cancer, esophageal cancer and contralateral BC. ${ }^{22,23}$ Conversely, hormonal therapy exhibited a protective effect against SPM occurrence, ${ }^{24}$ although patients treated with tamoxifen had an elevated risk of corpus uteri cancer. ${ }^{25}$ In addition, genetic alterations, particularly BRCA1/2 mutations, were proven to be risk factors for SPM occurrence. ${ }^{24,26}$ Despite massive efforts in exploring predictors of increased SPM risk, few studies have considered the effect of HER2 status. ${ }^{24}$

This study aimed to investigate the differential SPM occurrence after first primary BC according to HER2 status, using Surveillance, Epidemiology, and End Results (SEER) database. We used standardized incidence ratio (SIR) to profile and compare the SPM incidence of BC patients with different HER2 status. To adjust for competing death risk, subsequent competing risk analysis was conducted to explore the role of HER2 status in SPM risk. We further assessed the survival outcomes according to HER2 status and SPM occurrences.

\section{Materials and Methods}

\section{Study Population}

SEER*Stat software (version 8.3.9) was used to obtain the SEER 18 Registries (excluding AK) Research Data (2000-2018, based on November 2020 submission), which covers approximately $27.8 \%$ of the US population. From the cases reported to this database, we incorporated female patients older than 18 years old and diagnosed with malignant $\mathrm{BC}$. The malignant cases were identified based on behavior codes in ICD-O-3. Only cases with definite HER 2 and HR status diagnosed between 1 January, 2010 and 31 December, 2018 were selected, as SEER program did not collect HER2 status of BC before the year of 2010. Cases without positive histology or active follow-up were not included, as well as those death certificate or autopsy only cases. Besides, 7454 patients were excluded, because their survival months or follow-up times were shorter than 2 months or unknown. According to Warren and Gates criteria, ${ }^{27} \mathrm{SPM}$ after BC was defined as a subsequent, new primary cancer among $\mathrm{BC}$ survivors. To minimize the possibility of misdiagnosed recurrence or metastases, SEER registries classified multiple primary cancers based on the cancer site of origin, histology, tumor behavior, and laterality of paired organs. ${ }^{28}$ Moreover, new malignancy diagnosed within 2 months since the date of BC diagnosis were excluded in order to avoid synchronous cancers.

\section{Study Variables}

Patients were classified into 4 groups based on the diagnosis age: $<45,45-59,60-74, \geq 75$ years. Four-grade system was utilized to acquire $\mathrm{BC}$ grades according to microscopic examination of tumor tissue. A combined stage group was derived based on clinical and pathologic information composed of primary tumor (T), regional lymph nodes $(\mathrm{N})$ and distant metastasis (M). The TNM classification followed the TNM manuals published by the American Joint Committee on Cancer (AJCC) and the International Union Against Cancer (UICC), the most upto-date edition at that time. Hormone receptor (HR) status was obtained via the immunohistochemistry test of estrogen or progesterone receptor (ER/PR) ${ }^{29}$ HER2 status was defined via the algorithm for deriving HER2 summary variable, according to immunohistochemistry and in situ hybridization tests. ${ }^{30}$ The timing of the SPM and death occurrence and cause of death were also provided.

SPM incidence in first BC patient was quantified by SIR computed by SEER*Stat. SIR is the observed incidence of SPM among BC survivors to the expected incidence, ${ }^{31,32}$ based on specific cancer incidence rates for the general population in SEER 18 Registries (excluding AK). The 95\% CI for SIR was estimated assuming a Poisson distribution for the observed SPM numbers. SIRs of SPM following HER2+ and HER2-negative (HER2-) BC patients were acquired overall and stratified by HR status, diagnosis age and malignancy site.

\section{Statistical Analysis}

According to HER2 status of first $\mathrm{BC}$, patient characteristics, and most importantly, whether suffering from SPMs were analyzed. Pearson's chi-square test was employed to compare the variable distribution. 
Due to the natural different morbidity in different cohorts, SIRs reliably manifested SPM incidence in BC survivors. With the expected numbers as an offset, Poisson regression estimated the relative risk (RR) of HER2 status on SPM. Since latency after first $\mathrm{BC}$ diagnosis varied and a large proportion of patients died before SPM occurrence, competing risk analysis was adopted to verify the risk of SPM after HER2- and HER2+ BC. ${ }^{33,34}$ We utilized Cumulative incidence function (CIF) to describe the occurrence, and Gray's test to assess the statistical difference of occurrence probability. Subsequently, the hazard ratios (HRs) and $95 \%$ CIs of developing SPM after HER2+ vs HER2- BC were calculated using proportional subdistribution hazards regression, which adjusts for competing risk of death and other significant characteristics. Regarding survival, overall survival (OS) and breast cancer-specific survival (BCSS) were calculated from the date of diagnosis of $\mathrm{BC}$ to the date of death, due to any causes and $\mathrm{BC}$ specifically. Kaplan-Meier survival analyses were utilized to estimate the OS and BCSS of BC patients based on the presence or absence of SPMs, and their intrinsic HER2 status. Log rank tests were adopted to determine the difference significance.

All statistical analyses were performed using $\mathrm{R}$ version 4.0.5 software. Statistical significance was set at twosided, with $\mathrm{p}$ value $<0.05$ defined as statistically significant.

\section{Results}

\section{Patient Characteristics}

409,796 BC patients diagnosed between 2010 and 2018 were selected in this study, with a median follow-up period of 44 months. HER2+ BC $(\mathrm{N}=64,740)$ accounted for $15.80 \%$ of all patients, $3.54 \%$ of which $(\mathrm{N}=2293)$ were reported to develop SPMs, while HER2- BC ( $\mathrm{N}=345,056)$ had a higher SPM incidence of $4.63 \%(\mathrm{~N}=15990)$. Table 1 outlines the clinicopathologic characteristics and occurrence of SPM, grouped by HER2 status at first primary BC diagnosis. All factors were statistically significantly associated with HER2 status $(\mathrm{p}<0.001)$ based on Pearson's chi-square test. For HER2+ BC patients, 11,592 (17.91\%) women were diagnosed aged $<45$ and 26,282 (40.60\%) aged between 45 and 59, accounting for significantly higher proportions than HER2- BC patients.

Besides, HER2+ $\mathrm{BC}$ were more likely to have advanced grade and stage, as well as negative HR status. However, SPMs were remarkably less common in HER2 $+\mathrm{BC}$ patients, despite their clinicopathologic characteristics suggest more aggressive clinical courses.
Table I Baseline Characteristics of Invasive Breast Cancer Samples in 18 SEER Registries (Excluding AK), 2010-2018

\begin{tabular}{|c|c|c|c|}
\hline Characteristics & $\begin{array}{l}\text { HER2- } \\
\text { Positive }\end{array}$ & $\begin{array}{l}\text { HER2- } \\
\text { Negative }\end{array}$ & $p$-value \\
\hline & $64,740(100.00)$ & $345,056(100.00)$ & \\
\hline Age (years) & & & $<0.001 *$ \\
\hline$<45$ & $11,592(17.91)$ & 38,307 (11.10) & \\
\hline $45-59$ & $26,282(40.60)$ & $|17,29|(33.99)$ & \\
\hline $60-74$ & $19,906(30.75)$ & 133,339 (38.64) & \\
\hline$\geq 75$ & $6960(10.75)$ & $56,119(16.26)$ & \\
\hline Grade & & & $<0.001 *$ \\
\hline Well differentiated & $3030(4.68)$ & $88,356(25.61)$ & \\
\hline Moderately & $22,762(35.16)$ & 152,963 (44.33) & \\
\hline differentiated & & & \\
\hline Poorly differentiated/ & $35,310(54.54)$ & 91,326 (26.47) & \\
\hline Unknown & $3638(5.62)$ & $|2,4| \mid(3.60)$ & \\
\hline Stage & & & $<0.001 *$ \\
\hline $0 / 1$ & $24020(37.10)$ & $181,579(52.62)$ & \\
\hline II & 23874 (36.88) & 107,334 (31.1I) & \\
\hline III & $9788(15.12)$ & $34,492(10.00)$ & \\
\hline IV & 4997 (7.72) & 13,937 (4.04) & \\
\hline Unknown & 2061 (3.18) & $7714(2.24)$ & \\
\hline HR status & & & $<0.001 *$ \\
\hline Negative & 19,162 (29.6) & $45,84 \mid$ (I3.29) & \\
\hline Positive & 45,578 (70.4) & 299,215 (86.7I) & \\
\hline With SPM & & & $<0.001 *$ \\
\hline Yes & $2293(3.54)$ & $15,990(4.63)$ & \\
\hline No & $62,447(96.46)$ & 329,066 (95.37) & \\
\hline
\end{tabular}

Notes: * $p$-value $<0.05$ was statistically significant using Pearson's chi-square test. Abbreviations: HR, hormone receptor; HER2, human epidermal growth factor receptor 2 .

\section{Profile of SPM Incidences}

18,283 patients were diagnosed with at least one SPM after first BC, with 19,297 SPMs overall. It was significantly more than the 17,346.64 expected cases based on the rates in the general population (SIR $=1.11 ; 95 \% \mathrm{CI}$ 1.10-1.13). As listed in Table 2, compared with the general population, SPM incidence was only significantly elevated following HER2- BCs, of which SIR was 1.13 (95\% CI 1.11-1.14; $\mathrm{p}<0.05$ ). Based on the Poisson regression, the RR of SPMs following HER2+ versus HER2BCs was $0.92(95 \%$ CI $0.88-0.96 ; \mathrm{p}<0.001)$. The results were consistent in both HR status subgroups. Among BC molecular subtypes, the uppermost SIR of 1.30 (95\% CI 1.24-1.35) belongs to the triple negative BC. In different age subgroups, the significantly lower SPM incidence after HER2+ BCs was only consistent in patients older than 44 years. It is noteworthy that HER2+ BC patients aged 60 
Table 2 Standardized Incidence Ratios (SIRs) of Second Primary Malignancy (SPM) According to HER2 Status, Stratified by HR Status and Age at Breast Cancer Diagnosis

\begin{tabular}{|c|c|c|c|c|c|c|}
\hline & \multicolumn{2}{|c|}{ HER2+ } & \multicolumn{2}{|c|}{ HER2- } & \multicolumn{2}{|c|}{ HER2+ versus HER2- } \\
\hline & SIR & $95 \% \mathrm{Cl}$ & SIR & $95 \% \mathrm{Cl}$ & $\mathbf{R R}(95 \% \mathrm{Cl})$ & $p$-value \\
\hline All patients & 1.03 & $0.99-1.07$ & $1.13^{*}$ & I.II-I.I4 & $0.92(0.88-0.96)^{*}$ & $<0.001$ \\
\hline \multicolumn{7}{|l|}{ HR status } \\
\hline Positive & 1.02 & $0.98-1.07$ & $1.10 *$ & $1.09-1.12$ & $0.94(0.88-1.00)^{*}$ & $<0.001$ \\
\hline Negative & 1.04 & $0.97-1.12$ & $1.30 *$ & $1.24-1.35$ & $0.80(0.74-0.88)^{*}$ & $<0.001$ \\
\hline \multicolumn{7}{|l|}{ Age } \\
\hline$<45$ & $1.99 *$ & I.75-2.44 & $2.12^{*}$ & $1.99-2.25$ & $0.94(0.82-1.07)$ & 0.347 \\
\hline $45-59$ & $1.13^{*}$ & $1.06-1.21$ & $1.28 *$ & $1.25-1.32$ & $0.88(0.82-0.95)^{*}$ & $<0.001$ \\
\hline $60-74$ & $0.87^{*}$ & $0.8 \mathrm{I}-0.93$ & 1.01 & $0.99-1.04$ & $0.86(0.80-0.92)^{*}$ & $<0.001$ \\
\hline$\geq 75$ & 0.94 & $0.85-1.04$ & $1.07 *$ & I.04-I.I & $0.88(0.79-0.98)^{*}$ & 0.02 \\
\hline
\end{tabular}

Note: *p-value<0.05.

Abbreviations: SIR, standardized incidence ratio, $\mathrm{Cl}$, confidence interval, RR, relative risk.

74 had a significantly reduced SPM incidence than the general population (SIR, 0.87; 95\% CI 0.81-0.93).

The impact of HER2 status on SPM risk may differ by specific SPM types, thus SPM occurrence in different sites was then profiled. The most frequent SPM site was female breast $(\mathrm{N}=5499[28.50 \%])$, followed by lung and bronchus $(\mathrm{N}=2388$ [12.38\%]), colon ( $\mathrm{N}=1129$ [5.85\%]), corpus uteri $(\mathrm{N}=1090$ [5.65\%]) and thyroid $(\mathrm{N}=968$ [5.02\%]). The number of patients developing SPM and the SIRs of different SPM types following HER2+ vs HER2- BC are exhibited in Figure 1. For HER2+ BC patients, the value of SIRs significantly greater than 1 , were only observed in second thyroid cancer, renal carcinoma, leukemia and gastric cancer (site-specific SIRs were 2.38, 1.58, 1.69 and 1.51, respectively; all $\mathrm{p}<0.05$ ). Except gastric cancer, incidence of the other three SPMs in HER2- BC survivors

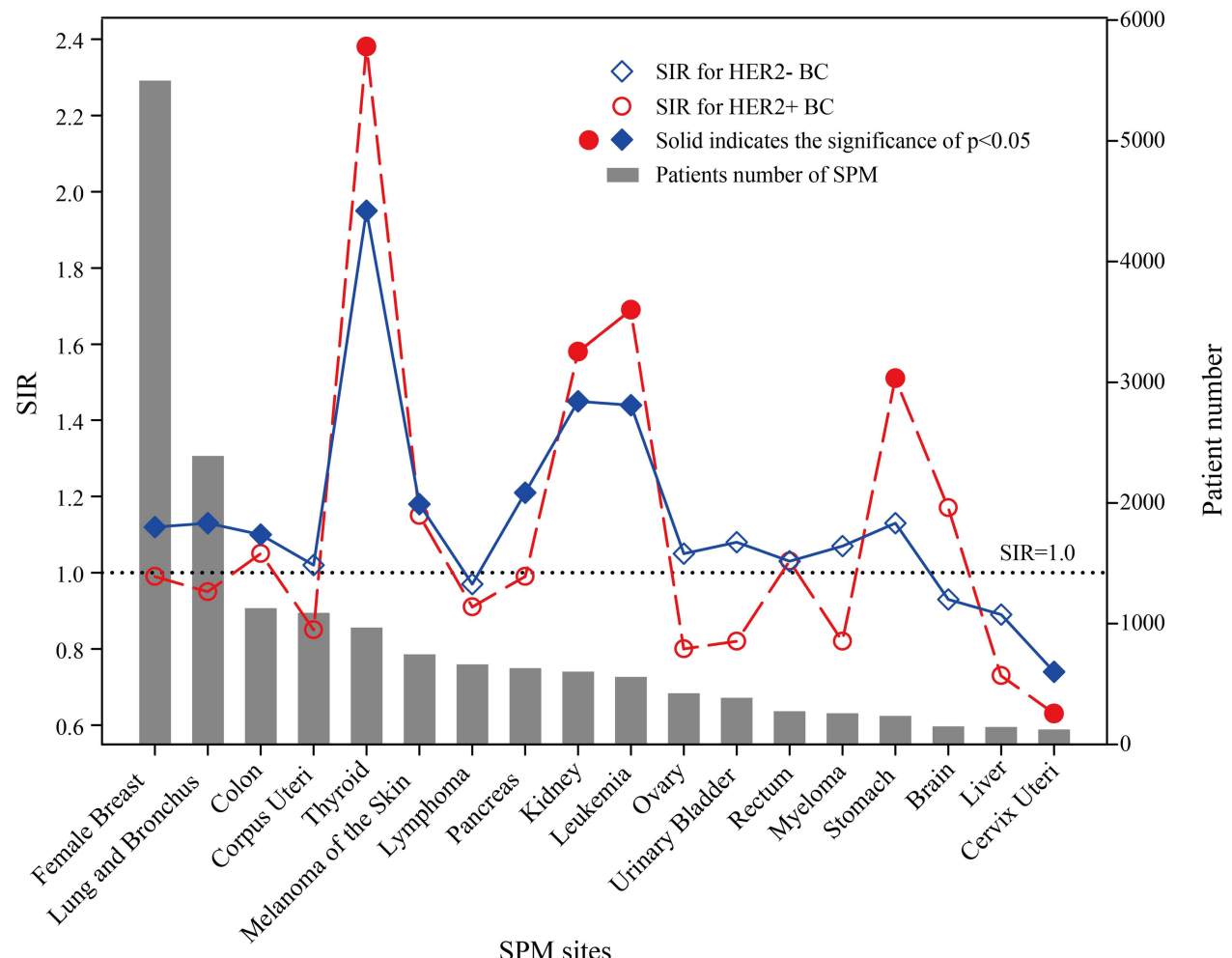

Figure I Standardized incidence ratios (SIRs) and patient numbers of second primary malignancies (SPM) in specific sites. 
also significantly exceed the general population. For most SPM sites, including female breast, lung and bronchus, colon, melanoma and pancreas, SPMs with higher occurrence rates than the general population were only observed among HER2- BC patients. Poisson regression was used again to estimate different second malignancy risks according to different HER2 status. Figure 2 demonstrated the forest plots, visualizing the relative risks of HER2 positive status for SPM occurrence. Notably, only for second primary malignancies in female breast (RR, $0.89 ; 95 \% \mathrm{CI}, 0.82-0.96 ; \mathrm{p}=0.003)$ and lung and bronchus (RR, 0.84; 95\% CI, 0.74-0.95; $\mathrm{p}=0.007$ ), the risks were significantly reduced after HER2+ BC in comparison to HER2- BC patients. The risk was marginally reduced for second corpus uteri cancer (RR, 0.83 ; 95\% CI, 0.69 $0.99 ; \mathrm{p}=0.041)$. In addition, HER2 positive status was revealed to be a risk factor for second thyroid malignancy occurrence (RR, 1.22; 95\% CI, 1.04-1.42; $\mathrm{p}=0.015$ ).

\section{Competing Risk Analysis}

When estimating the SPM incidence, occurrence of death can compete and should be taken into account by competing risk analysis. Figure 3 depicted the cumulative incidence of SPMs and deaths via CIF. The 5-year cumulative incidence of SPMs following first HER2- and HER2+ BC were $5.16 \%$ and $4.09 \%$, respectively. HER2- BC patients had a markedly higher cumulative SPM incidence than HER2 + BC patients $(\mathrm{p}<0.001)$, and on the contrary, significantly lower death incidence $(\mathrm{p}<0.001)$. To understand whether the difference of SPM cumulative incidence is solely due to the HER2 status, subdistribution hazard function was performed, as shown in Table 3. Univariate regression analysis indicated that not only HER 2 status but also age, grade, stage and HR status were correlated with SPM risk. Multivariate regression analysis further screened HER2 status and diagnosis age as independent risk factors for SPM. HER2 positive status significantly reduced the overall SPM risk (sdHR, 0.86; 95\% CI, 0.82 $0.90 ; \mathrm{p}<0.001)$, in accordance with aforementioned results. However, the inconsistency was that SPM risk increased as patients diagnosed at older age $(\mathrm{p}<0.001)$, and $\mathrm{HR}$ status did not differ statistically in SPM risk $(\mathrm{p}=0.28)$.

In addition, multivariate proportional subdistribution hazards regression was performed according to different SPM sites. For second primary BC, as well as lung and bronchus cancer, SPM risk decreased significantly after first HER2+ BC, with sdHR of 0.82 (95\% CI, 0.75-0.89; $\mathrm{p}<0.001)$ and $0.78 \quad(95 \% \quad$ CI $\quad 0.68-0.90 ; \quad \mathrm{p}<0.001)$,

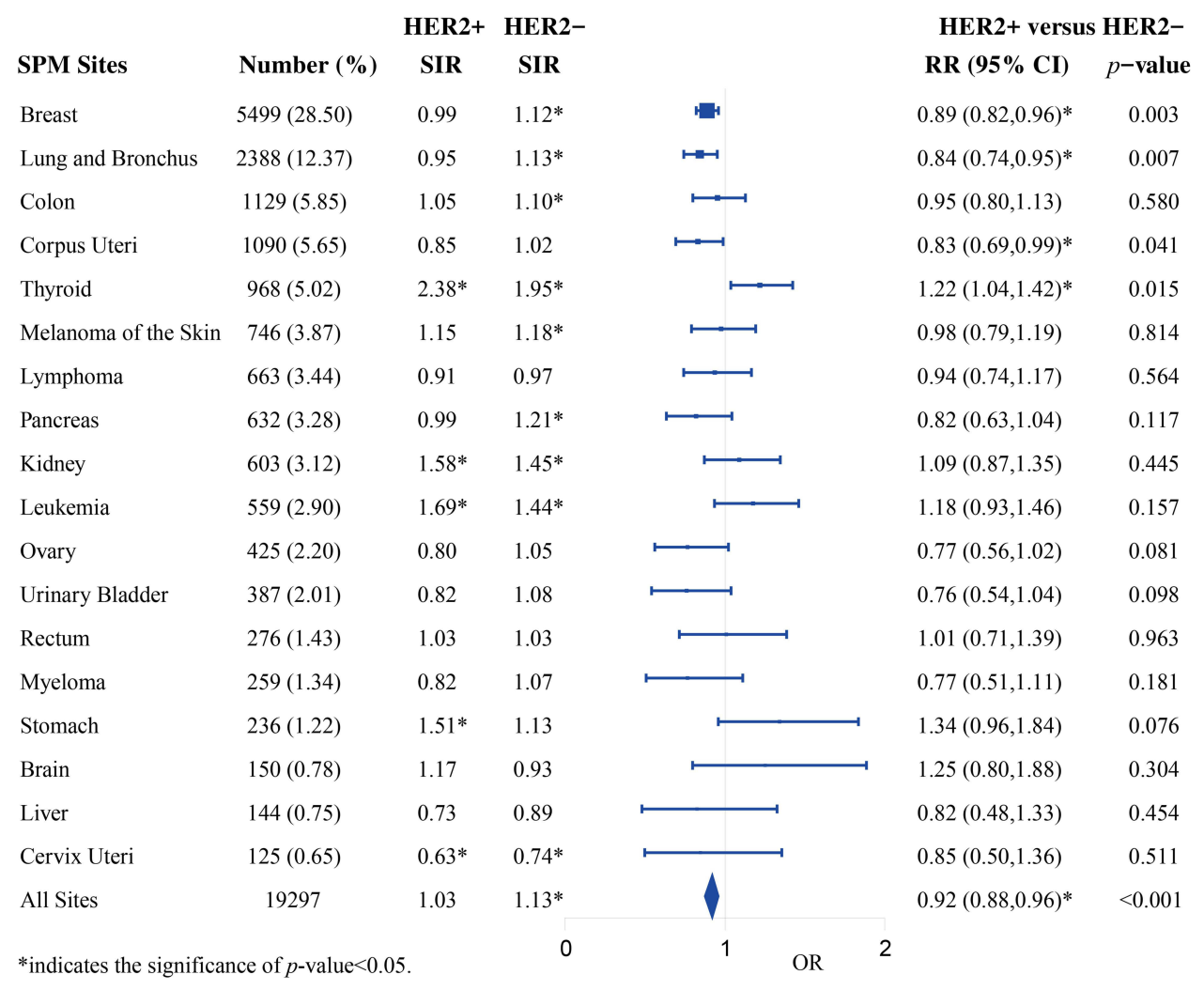

Figure 2 Site-specific Standardized incidence ratios (SIRs) and relative risks of developing second primary malignancies (SPM) after HER2+/HER2- BCs. 


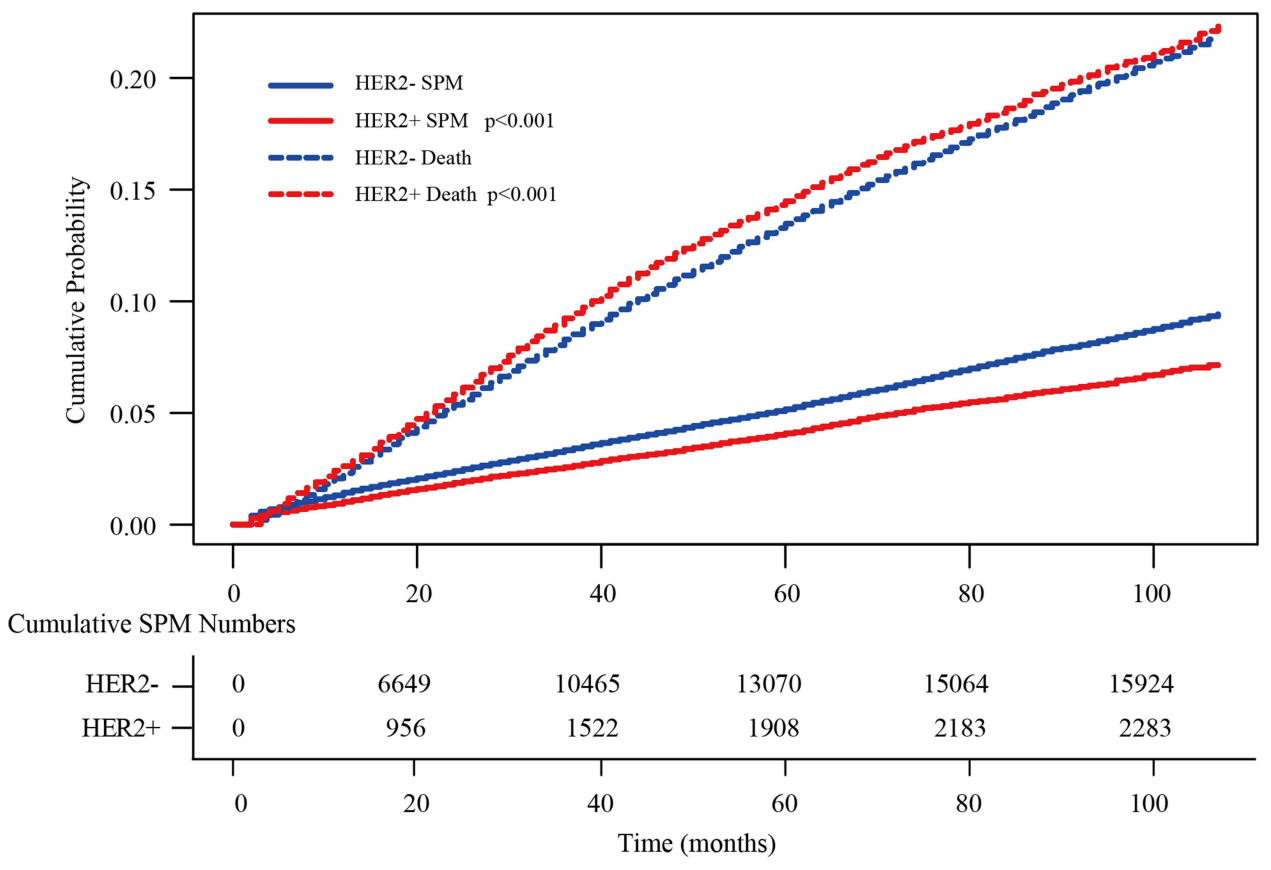

Figure 3 Cumulative incidence curves for second primary malignancies (SPM) and deaths in HER2+ and HER2- breast cancer patients.

respectively. Whereas, little variance on second corpus uteri cancer risk were shown between $\mathrm{BC}$ patients with different HER2 status (sdHR, 0.79; 95\% CI, 0.66-0.95; $\mathrm{p}=0.014$ ), and no difference on second thyroid cancer risk (sdHR, 1.18; 95\% CI, 1.00-1.39; $\mathrm{p}=0.053$ ).

\section{Kaplan-Meier Survival Analysis}

Finally, the impact of SPM and HER2 status on OS and BCSS was analyzed (Figure 4). Among the 18,283 BC patients with SPMs, 1828 (10.00\%) died of BC, whereas 3645 (19.94\%) patients died of other causes. In line with previous studies, SPM was associated with prominently worse OS (HR, 2.00; 95\% CI, 1.93-2.08; $\mathrm{p}<0.0001$ ), but slightly worse BCSS (HR, 1.09; 95\% CI, 1.03-1.14; $\mathrm{p}=0.0012)$. The 5 -year OS probability was $74.4 \%$ $(\mathrm{p}=0.004)$ and $85.5 \%(\mathrm{p}<0.001)$ for $\mathrm{BC}$ patients with and without SPMs, respectively. We also found a substantial difference in BCSS between HER2- and HER2+ BC patients (HR, 0.79; 95\% CI, 0.77-0.82; $<<0.0001$ ), but a minor disparity in OS (HR, 0.97; 95\% CI, 0.95-1.00; $\mathrm{p}=0.0221$ ). Better survival outcomes favored negative HER2 status. For BC patients in the existence of SPMs, reversely, positive HER2 status was associated with superior OS (5-year OS, $76.2 \%$ vs $74.2 \% ; \mathrm{p}=0.0011$ ). However, no variance was shown regarding BCSS, indicating the survival difference may be attributed to other causes of death concerning SPM.

\section{Discussion}

Our research mainly focused on the association between SPM occurrence and HER2 status of first BC. According to the SIRs and Poisson regression results, significantly increased incidences of SPMs were demonstrated in HER2- BC patients. This was remarkable for second primary $\mathrm{BC}$ and lung cancer. Competing risk analysis also indicated HER2 negative status as an independent risk factor for SPM. In 2015, Marcheselli et al reported that HER2 positivity was associated with increased risk of secondary digestive system and thyroid cancer. ${ }^{24}$ Incorporating 305 cases and 1525 control patients, this study was limited by small sample sizes. In our study, for the first time, two different methods were performed to explore the risk factors for SPM. Older age is associated with intrinsic incremental cancer risk. And HER2 status varied with age according to our baseline characteristics analysis. Therefore, SPM risk and effect of HER2 status were quantified using SIRs and Poisson regression, by introducing age-specific rates in the general population as external reference. We further performed competing risk analysis to verify the impacts of risk factors on SPM occurrence, considering death as a competing risk. Eventually, two approaches yielded similar result that remarkable SPM risk difference existed between HER2+ and HER2- BC patients. The SPM risk increased when $\mathrm{BC}$ patient was diagnosed at older age. 
Table 3 Univariate and Multivariate Proportional Subdistribution Hazard Regression for Second Primary Malignancy (SPM) in Breast Cancer Patients

\begin{tabular}{|c|c|c|c|c|}
\hline \multirow[b]{2}{*}{ Characteristics } & \multicolumn{2}{|c|}{ Univariate } & \multicolumn{2}{|c|}{ Multivariate } \\
\hline & sdHR (95\% Cls) & $p$-value & sdHR (95\% Cls) & $p$-value \\
\hline \multicolumn{5}{|l|}{ Age (years) } \\
\hline$<45$ & Reference & & Reference & \\
\hline $45-59$ & $\mathrm{I} .42(\mathrm{I} .33, \mathrm{I} .5 \mathrm{I})^{*}$ & $<0.001$ & $\mathrm{I} .40(\mathrm{I} .3 \mathrm{I}, \mathrm{I} .49)^{*}$ & $<0.001$ \\
\hline $60-74$ & $2.15(2.02,2.28)^{*}$ & $<0.001$ & $2.09(1.96,2.22)^{*}$ & $<0.001$ \\
\hline$\geq 75$ & $2.56(2.39,2.74)^{*}$ & $<0.001$ & $2.49(2.32,2.67)^{*}$ & $<0.001$ \\
\hline \multicolumn{5}{|l|}{ Grade } \\
\hline Well differentiated & Reference & & Reference & \\
\hline Moderately differentiated & $0.93(0.90,0.97)^{*}$ & $<0.001$ & $0.98(0.94,1.02)$ & 0.29 \\
\hline Poorly differentiated/Undifferentiated & $0.81(0.77,0.84)^{*}$ & $<0.001$ & $0.94(0.89,0.98)^{*}$ & 0.0043 \\
\hline Unknown & $0.80(0.74,0.87)^{*}$ & $<0.001$ & $0.91(0.84,0.99)^{*}$ & 0.024 \\
\hline \multicolumn{5}{|l|}{ Stage } \\
\hline $0 / 1$ & Reference & & Reference & \\
\hline II & $0.91(0.88,0.94)^{*}$ & $<0.001$ & $0.98(0.95,1.02)$ & 0.31 \\
\hline III & $0.91(0.87,0.96)^{*}$ & $<0.001$ & $1.03(0.97,1.08)$ & 0.36 \\
\hline IV & $0.67(0.6 \mathrm{I}, 0.74)^{*}$ & $<0.001$ & $0.73(0.66,0.80)^{*}$ & $<0.001$ \\
\hline Unknown & $0.83(0.74,0.93)^{*}$ & 0.0016 & $0.85(0.76,0.95)^{*}$ & 0.005 \\
\hline \multicolumn{5}{|l|}{ HR status } \\
\hline Negative & Reference & & Reference & \\
\hline Positive & I.II (I.06, I.I5)* & $<0.001$ & $0.98(0.93,1.02)$ & 0.28 \\
\hline \multicolumn{5}{|l|}{ HER2 status } \\
\hline Negative & Reference & & Reference & \\
\hline Positive & $0.77(0.74,0.80)^{*}$ & $<0.001$ & $0.86(0.82,0.90)^{*}$ & $<0.001$ \\
\hline
\end{tabular}

Note: *p-value<0.05.

Abbreviations: sdHR, subdistribution hazard ratios; HR, hormone receptor; HER2, human epidermal growth factor receptor 2.

Although HER2- BC was not so aggressive as HER2 $+\mathrm{BC}$, the higher incidence of SPM makes it a new concern for $\mathrm{BC}$ survivors. As mentioned above, compared with HER2+ BC, HER2- BC patients had superior BCSS in all patients, but worse OS in patients followed by SPM. To improve the OS of HER2- BC patients, it is important to find out the potential contributing factors that are relevant to HER2 status.

In recent decades, HER2 has been an established target in BC therapy. Biologically, HER2 exists as a driving factor in various tumor types derived from epithelia, represented by $\mathrm{BC}$, non-small-cell lung cancer, ${ }^{35,36}$ gastric and gastroesophageal junction cancers. ${ }^{37,38}$ It was further confirmed by the fact that HER2 overexpression or gene amplification were detected in a variety of human malignancies including lung, ${ }^{39}$ gastric, ${ }^{40}$ ovarian, ${ }^{41}$ biliary tract ${ }^{42}$ and colorectal cancers. ${ }^{43}$ In therapeutic application, anti-HER2 therapies have already shown efficacy in HER2-positive advanced gastric cancers. ${ }^{37,44,45}$ Therefore, we speculated that when HER2+ patients undertook anti-HER2 treatment for $\mathrm{BC}$, these targeting therapies would probably simultaneously extinguish and eliminate latent HER2+ transformed cells in other system. As a result, the SPM incidence decreased after HER2+ BC due to the HER2targeted treatment. It is known that more than two decades have passed since the approval of the first anti-HER2 targeted therapy, trastuzumab by FDA in 1998. And HER2-targeted treatment has become the standard of care in managing HER2+ BC patients all over the world. Hence, recent calendar year and HER2 positivity diagnosis have been used as a crude proxy to support this hypothesis. Besides anti-HER2 therapy, HER2+ BC patients were more likely to received adjuvant chemotherapy, ${ }^{46,47}$ which may also contribute to the declining SPM incidences. 

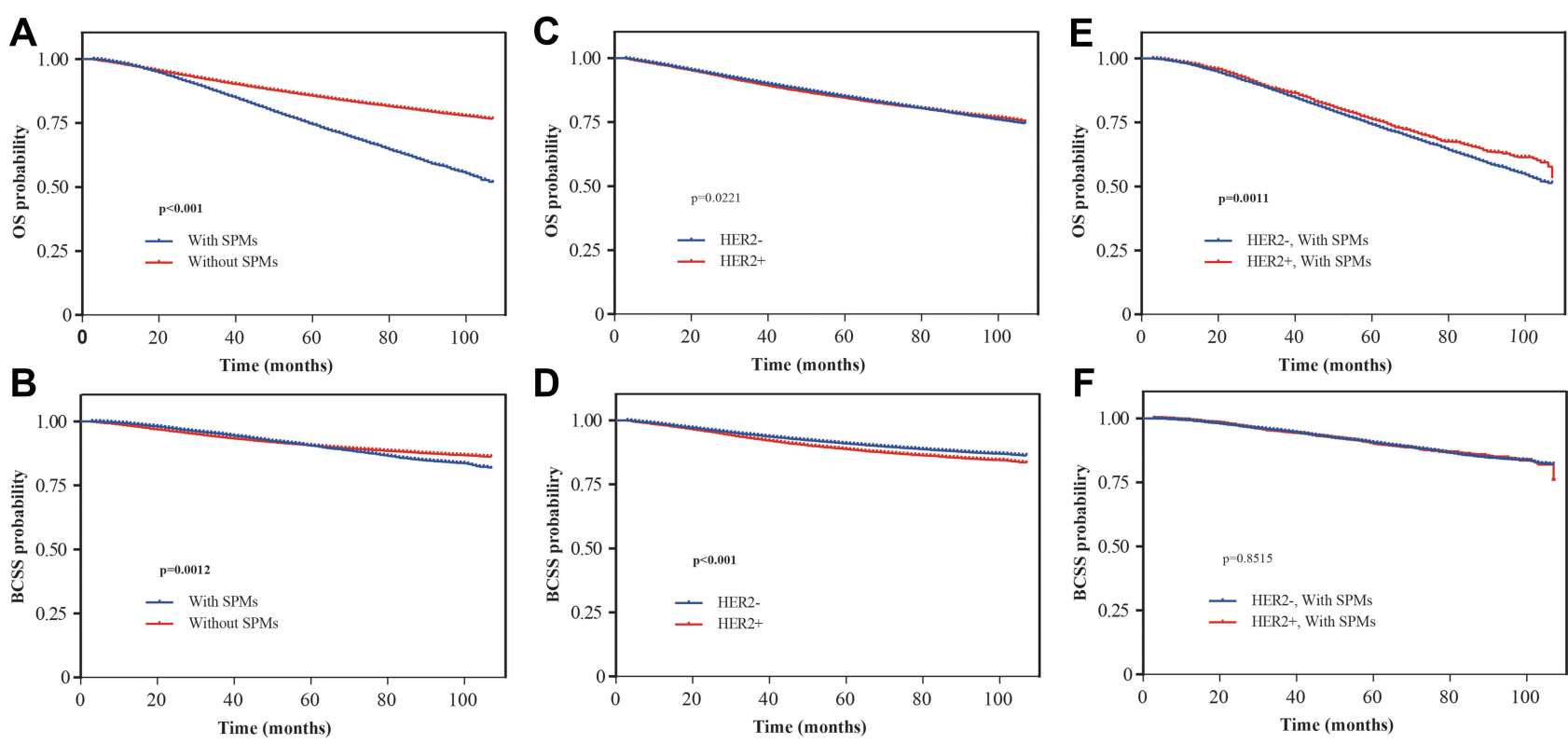

Figure 4 Survival curves of OS and BCSS based on (A and B) SPM occurrence; (C and D) HER2 status in all patients and (E and F) HER2 status in patients with SPMs.

As for the intrinsic risk factors for SPM, shared genetic predisposition associated with BC HER2 status is inevitable. It has been reported that pathogenic germline variants were exhibited in $8 \%$ of adult cancer cases. ${ }^{48}$ Genomic alterations were also reported to be incriminated towards HER2+ BC. ${ }^{49,50}$ Moreover, several genes such as FCRLM1, BLK and IHGD were indicative of the development of SPMs among BC patients. $^{49,50}$ It led to the assumption that some unique intrinsic genetic variants existed in $\mathrm{BC}$ patients may be a natural inclination for the SPMs. Indirect support for the hypothesis can be gleaned from the findings that higher proportion of HER2 positive status was observed in $\mathrm{BC}$ patients carrying BRCA2 mutations, meanwhile, BRCA2 carriers were less likely to be diagnosed with SPMs. ${ }^{51}$ In summary, the SPM risk reduction after HER2+ BC may be attributable to the widespread antiHER2 treatment combined with chemotherapy, as well as genetic susceptibility. More investigation about therapeutic and inherent factors should be conducted to reveal the disparity between different BC HER2 status.

The differential SPM occurrence following HER2+ and HER2- BC was comprehensively profiled in this context. It was based on a large cohort from SEER program, thus reducing the sampling error and ensuring the quality of the data. However, there were still some limitations. First, some metastases and relapses may be mistaken as SPM. Although SPMs were strictly screened out according to Warren and Gates criteria including histopathologic confirmation, definitive evidence of cancer biologically independence could not be provided. Second, because of data incompleteness, we could not analyze effects of adjuvant treatment, especially anti-HER2 therapies. Third, some potential risk factors were not available, such as smoking, alcohol use and body mass index. More researches with detailed information on treatment delivery, genetic variants and other confounders are needed to reveal the disparity.

\section{Conclusion}

SPM incidence significantly declined after HER $2+$ BC, demonstrated by different quantitative tools including SIR and competing risk model. The OS deterioration in HER2- BC patients accompanied by SPM indicated the requirement to explain this disparity, with the aim of reducing SPM risk.

\section{Data Sharing Statement}

The data analyzed in this study can be obtained from the corresponding author under reasonable request.

\section{Ethics Approval}

This study was reviewed and approved by the Institutional Review Board of Guangdong Provincial People's Hospital.

\section{Consent for Publication}

Consent for publication was obtained from all authors. 


\section{Acknowledgments}

The authors acknowledge the efforts of the Surveillance, Epidemiology and End Results (SEER) Program tumor registries in providing detailed information.

\section{Author Contributions}

All authors made a significant contribution to the work reported, whether that is in the conception, study design, execution, acquisition of data, analysis and interpretation, or in all these areas; took part in drafting, revising or critically reviewing the article; gave final approval of the version to be published; have agreed on the journal to which the article has been submitted; and agree to be accountable for all aspects of the work.

\section{Funding}

This study was funded by Guangzhou Municipal Science and Technology Project (201804010430) and Natural Science Foundation of Guangdong Province (2018A030313292).

\section{Disclosure}

The authors declare no conflicts of interest in this work.

\section{References}

1. Siegel RL, Miller KD, Fuchs HE, Jemal A. Cancer Statistics, 2021. CA Cancer J Clin. 2021;71(1):7-33. doi:10.3322/caac.21654

2. Waks AG, Winer EP. Breast cancer treatment: a review. JAMA. 2019;321(3):288-300

3. Allemani C, Matsuda T, Di Carlo V, et al. Global surveillance of trends in cancer survival 2000-14 (Concord-3): analysis of individual records for 37513025 patients diagnosed with one of 18 cancers from 322 population-based registries in 71 countries. Lancet. 2018;391 (10125):1023-1075.

4. Kim MJ, Ro JY, Ahn SH, Kim HH, Kim SB, Gong G. Clinicopathologic significance of the basal-like subtype of breast cancer: a comparison with hormone receptor and Her2/neu-overexpressing phenotypes. Hum Pathol. 2006;37(9):1217-1226.

5. Wolff AC, Hammond ME, Schwartz JN, et al. American Society of Clinical Oncology/College of American Pathologists guideline recommendations for human epidermal growth factor receptor 2 testing in breast cancer. J Clin Oncol. 2007;25(1):118-145.

6. Gutierrez C, Schiff R. HER2: biology, detection, and clinical implications. Arch Pathol Lab Med. 2011;135(1):55-62.

7. Cameron D, Piccart-Gebhart M, Gelber R, et al. 11 years' follow-up of trastuzumab after adjuvant chemotherapy in HER2-positive early breast cancer: final analysis of the HERceptin Adjuvant (HERA) trial. Lancet (London, England). 2017;389(10075):1195-1205.

8. Perez EA, Romond EH, Suman VJ, et al. Trastuzumab plus adjuvant chemotherapy for human epidermal growth factor receptor 2-positive breast cancer: planned joint analysis of overall survival from NSABP B-31 and NCCTG N9831. J Clin Oncol. 2014;32(33):3744-3752.

9. Rimawi MF, Schiff R, Osborne CK. Targeting HER2 for the treatment of breast cancer. Annu Rev Med. 2015;66(1):111-128.
10. Molina-Montes E, Requena M, Sanchez-Cantalejo E, et al. Risk of second cancers cancer after a first primary breast cancer: a systematic review and meta-analysis. Gynecol Oncol. 2015;136 (1):158-171.

11. Li Z, Wang K, Shi Y, Zhang X, Wen J. Incidence of second primary malignancy after breast cancer and related risk factors-Is breast-conserving surgery safe? A nested case-control study. Int $J$ Cancer. 2020;146(2):352-362.

12. Early Breast Cancer Trialists' Collaborative Group (EBCTCG). Effects of radiotherapy and of differences in the extent of surgery for early breast cancer on local recurrence and 15-year survival: an overview of the randomised trials. Lancet. 2005;366 (9503):2087-2106.

13. Katuwal S, Jousilahti P, Pukkala E. Causes of death among women with breast cancer: a follow-up study of 50481 women with breast cancer in Finland. Int J Cancer. 2021. doi:10.1002/ijc.33607

14. Rusner C, Wolf K, Bandemer-Greulich U, et al. Risk of contralateral second primary breast cancer according to hormone receptor status in Germany. Breast Cancer Res. 2014;16(5):452.

15. Raymond JS, Hogue CJ. Multiple primary tumours in women following breast cancer, 1973-2000. Br J Cancer. 2006;94(11):1745-1750.

16. Qian X, Jia H, Zhang Y, Ma B, Qin G, Wu Z. Risk factors and prediction of second primary cancer in primary female non-metastatic breast cancer survivors. Aging. 2020;12(19):19628-19640.

17. Schonfeld SJ, Curtis RE, Anderson WF, Berrington de González A. The risk of a second primary lung cancer after a first invasive breast cancer according to estrogen receptor status. Cancer Causes Control. 2012;23(10):1721-1728.

18. Li D, Weng S, Zhong C, et al. Risk of second primary cancers among long-term survivors of breast cancer. Front Oncol. 2019;9:1426.

19. Wei JL, Jiang YZ, Shao ZM. Survival and chemotherapy-related risk of second primary malignancy in breast cancer patients: a SEER-based study. Int J Clin Oncol. 2019;24(8):934-940.

20. Jeyakumar A, Chua T, Lam A, Gopalan V. The melanoma and breast cancer association: an overview of their 'second primary cancers' and the epidemiological, genetic and biological correlations. Crit Rev Oncol Hematol. 2020;152:102989.

21. Wei J, Jiang Y, Shao Z. Survival and chemotherapy-related risk of second primary malignancy in breast cancer patients: a SEER-based study. Int J Clin Oncol. 2019;24(8):934-940.

22. Burt LM, Ying J, Poppe MM, Suneja G, Gaffney DK. Risk of secondary malignancies after radiation therapy for breast cancer: comprehensive results. Breast. 2017;35:122-129.

23. Schaapveld M, Visser O, Louwman M, et al. Risk of new primary nonbreast cancers after breast cancer treatment: a Dutch population-based study. J Clin Oncol. 2008;26(8):1239-1246.

24. Marcheselli R, Marcheselli L, Cortesi L, et al. Risk of second primary malignancy in breast cancer survivors: a nested population-based case-control study. J Breast Cancer. 2015;18(4):378-385.

25. Andersson M, Jensen M, Engholm G, Henrik Storm H. Risk of second primary cancer among patients with early operable breast cancer registered or randomised in Danish Breast Cancer cooperative Group (DBCG) protocols of the 77, 82 and 89 programmes during 1977-2001. Acta Oncologica (Stockholm, Sweden). 2008;47(4):755-764.

26. Agarwal S, Pappas L, Matsen C, Agarwal J. Second primary breast cancer after unilateral mastectomy alone or with contralateral prophylactic mastectomy. Cancer Med. 2020;9(21):8043-8052.

27. Warren S, Gates O. Multiple primary malignant tumors. A survey of the literature and a statistical study. Am $J$ Cancer. 1932;16 (1):1358-1414.

28. Curtis RE, Freedman DM, Ries LAG, et al. Methods. In: Curtis RE, Freedman DM, Ron E, editors. New Malignancies Among Cancer Survivors: SEER Cancer Registries, 1973-2000. Bethesda, MD: National Cancer Institute. NIH Publ. No. 05-5302; 2006:9-14. 
29. Hammond M, Hayes D, Dowsett M, et al. American Society of Clinical Oncology/College of American Pathologists guideline recommendations for immunohistochemical testing of estrogen and progesterone receptors in breast cancer. Arch Pathol Lab Med. 2010;134(6):907-922.

30. Wolff A, Hammond M, Allison K, et al. Human Epidermal Growth Factor Receptor 2 testing in breast cancer: American Society of Clinical Oncology/College of American pathologists clinical practice guideline focused update. J Clin Oncol. 2018;36(20):2105-2122.

31. Liddell F. Simple exact analysis of the standardised mortality ratio. J Epidemiol Community Health. 1984;38(1):85-88.

32. Yasui Y, Liu Y, Neglia J, et al. A methodological issue in the analysis of second-primary cancer incidence in long-term survivors of childhood cancers. Am J Epidemiol. 2003;158(11):1108-1113.

33. Kim HT. Cumulative incidence in competing risks data and competing risks regression analysis. Clin Cancer Res. 2007;13(2 Pt 1):559-565.

34. Scrucca L, Santucci A, Aversa F. Regression modeling of competing risk using R: an in depth guide for clinicians. Bone Marrow Transplant. 2010;45(9):1388-1395.

35. Hotta K, Yanai H, Ohashi K, et al. Pilot evaluation of a HER2 testing in non-small-cell lung cancer. J Clin Pathol. 2020;73 (6):353-357.

36. Kuyama S, Hotta $\mathrm{K}$, Tabata $M$, et al. Impact of HER2 gene and protein status on the treatment outcome of cisplatin-based chemoradiotherapy for locally advanced non-small cell lung cancer. $J$ Thor Oncol. 2008;3(5):477-482.

37. Bang Y-J, Van Cutsem E, Feyereislova A, et al. Trastuzumab in combination with chemotherapy versus chemotherapy alone for treatment of HER2-positive advanced gastric or gastro-oesophageal junction cancer (ToGA): a Phase 3, open-label, randomised controlled trial. Lancet. 2010;376(9742):687-697.

38. Tanner M, Hollmen M, Junttila TT, et al. Amplification of HER-2 in gastric carcinoma: association with Topoisomerase IIalpha gene amplification, intestinal type, poor prognosis and sensitivity to trastuzumab. Ann Oncol. 2005;16(2):273-278.
39. Pellegrini C, Falleni M, Marchetti A, et al. HER-2/Neu alterations in non-small cell lung cancer: a comprehensive evaluation by real time reverse transcription-PCR, fluorescence in situ hybridization, and immunohistochemistry. Clin Cancer Res. 2003;9:3645-3652.

40. Abrahao-Machado L, Scapulatempo-Neto C. HER2 testing in gastric cancer: an update. World J Gastroenterol. 2016;22(19):4619-4625.

41. Slamon D, Godolphin W, Jones L, et al. Studies of the HER-2/neu proto-oncogene in human breast and ovarian cancer. Science (New York, $N$ Y). 1989;244(4905):707-712.

42. Nam A, Kim J, Cha Y, et al. Therapeutic implication of HER2 in advanced biliary tract cancer. Oncotarget. 2016;7(36):58007-58021.

43. Siena S, Sartore-Bianchi A, Marsoni S, et al. Targeting the human epidermal growth factor receptor 2 (HER2) oncogene in colorectal cancer. Ann Oncol. 2018;29(5):1108-1119.

44. Patel TH, Cecchini M. Targeted therapies in advanced gastric cancer. Curr Treat Options Oncol. 2020;21(9):70.

45. Oh DY, Bang YJ. HER2-targeted therapies - a role beyond breast cancer. Nat Rev Clin Oncol. 2020;17(1):33-48.

46. Romond E, Perez E, Bryant J, et al. Trastuzumab plus adjuvant chemotherapy for operable HER2-positive breast cancer. $N$ Engl $J$ Med. 2005;353(16):1673-1684.

47. Swain S, Baselga J, Kim S, et al. Pertuzumab, trastuzumab, and docetaxel in HER2-positive metastatic breast cancer. $N$ Engl J Med. 2015;372(8):724-734.

48. Huang KL, Mashl RJ, Wu Y, et al. Pathogenic germline variants in 10,389 adult cancers. Cell. 2018;173(2):355e314-370e314.

49. Armakolas A, Stathopoulos G, Nezos A, Theos A, Stathaki M, Koutsilieris M. Subdivision of molecularly-classified groups by new gene signatures in breast cancer patients. Oncol Rep. 2012;28 (6):2255-2263.

50. Stathopoulos GP, Armakolas A. Differences in gene expression between individuals with multiple primary and single primary malignancies. Int J Mol Med. 2009;24(5):613-622.

51. Fountzilas E, Konstantopoulou I, Vagena A, et al. Pathology of BRCA1- and BRCA2-associated breast cancers: known and less known connections. Clin Breast Cancer. 2020;20(2):152-159.
International Journal of General Medicine

\section{Publish your work in this journal}

The International Journal of General Medicine is an international, peer-reviewed open-access journal that focuses on general and internal medicine, pathogenesis, epidemiology, diagnosis, monitoring and treatment protocols. The journal is characterized by the rapid reporting of reviews, original research and clinical studies across all disease areas. The manuscript management system is completely online and includes a very quick and fair peer-review system, which is all easy to use. Visit http://www.dovepress.com/ testimonials.php to read real quotes from published authors. 